\title{
Double coset construction of moduli space of holomorphic bundles and Hitchin systems.
}

\author{
A.Levin円 †, M.Olshanetsky $]^{\ddagger}$, \\ Max-Planck-Institut für Mathematik, Bonn

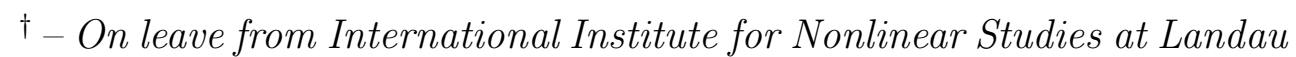 \\ Inst, Vorob'iovskoe sch. 2, Moscow, 117940, Russia \\ $\ddagger$ - On leave from ITEP, Bol.Cheremushkinskaya, 25, Moscow, 117 259, \\ Russia
}

\footnotetext{
${ }^{1}$ E-mail address: alevin@mpim-bonn.mpg.de

${ }^{2}$ E-mail address: olshanez@vxdesy.desy.de
} 
August 13, 2018

\begin{abstract}
We present a description of the moduli space of holomorphic vector bundles over Riemann curves as a double coset space which is differ from the standard loop group construction. Our approach is based on equivalent definitions of holomorphic bundles, based on the transition maps or on the first order differential operators. Using this approach we present two independent derivations of the Hitchin integrable systems. We define a "superfree" upstairs systems from which Hitchin systems are obtained by three step hamiltonian reductions. A special attention is being given on the Schottky parameterization of curves.
\end{abstract}

\title{
1 Introduction
}

The moduli space of holomorphic vector bundles over Riemann surfaces are popular subject in algebraic geometry and number theory. In mathematical physics they were investigated due to relations with the Yang-Mills theory [1] and the Wess-Zumino-Witten theory [2, 3]. The conformal blocks in the WZW theory satisfy the Ward identities which take a form of differential equations on the moduli space [4, 河. In this approach the moduli space is described as a double coset space of a loop group defined on a small circle on a Riemann surface [6].

The main goal of the paper is an alternative description of the moduli space and the Hitchin integrable systems [7] based on this construction. We start with a special group valued field on a Riemann surface which is defined as a map from a holomorphic basis in a vector bundle to a $C^{\infty}$ basis. This field is an analogous of the tetrade field in the General Relativity and we call it the Generalized Tetrade Field (GTF). The holomorphic structures can be extracted from GTF. They are described via the holomorphic transition maps, or by means of the operators $d^{\prime \prime}$. The former are invariant under the action of the global $C^{\infty}$ transformations, while the later under the action of the local holomorphic transformations. It allows to define the moduli space as a double coset space of GTF with respect to the actions of the local holomorphic transformations and the global $C^{\infty}$ transformations.

We introduce a cotangent bundle to GTF and invariant symplectic structure on it. The cotangent bundle to the moduli of holomorphic bundles can be obtained by the symplectic factorizations over the action of two types of commuting gauge transformations. This cotangent bundle is a phase space of the Hitchin integrable systems [7]. The tetrade fields in their turns are sections of the principle bundle over the Riemann surface, which satisfy some constraints equations. We interpret them as moment constraints in a big "superfree 
system" with a special gauge symmetry. This space is a cotangent bundle to the principle bundle. Thus the Hitchin systems are obtained by the three step symplectic reductions from this space.

We investigate specially our reductions in terms of Schottky parameterization, which is a particular case of the general construction. This parameterization was used to derived the Knizhnik-Zamolodchikov-Bernard equations on the higher genus curves [3, 8, 9]. On the other hand the quantum second order Hitchin Hamiltonians coincide with them on the critical level.

\section{Moduli of holomorphic vector bundles}

Let $\Sigma=\Sigma_{g}$ be a nondegenerate Riemann curve of genus $g$ with $g>1$. We will consider in this section a set of stable holomorphic structures on complex vector bundles over $\Sigma$ [1]. To define them we proceed in two ways based on the Cech and the Dolbeault cohomologies. Eventually, we come to the moduli space $\mathcal{L}$ of stable holomorphic bundles over $\Sigma_{g}$ and represent them as a double coset space (Proposition 2.3).

1. Consider a vector bundle $V$ over $\Sigma_{g}$. To be more concrete we assume that the structure group of $V$ is $G L(N, \mathbf{C})$. Let $\mathcal{U}_{a}, a=1, \ldots$ be a covering of $\Sigma_{g}$ by open subsets. We consider two bases in $V$ the holomorphic $\left\{e^{\text {hol }}\right\}$ basis and the smooth $C^{\infty}\left\{e^{C^{\infty}}\right\}$ one. In local coordinates $\left(z_{a} \in \mathcal{U}_{a}\right)$

$$
e_{a}^{h o l}=e^{h o l}\left(z_{a}\right), e_{a}^{C^{\infty}}=e^{C^{\infty}}\left(z_{a}, \bar{z}_{a}\right) .
$$

Let $h$ be the transition map between them $h_{a}=h\left(z_{a}, \bar{z}_{a}\right)$. Then locally in $\mathcal{U}_{a}$ we have

$$
h_{a} e_{a}^{C^{\infty}}=e_{a}^{h o l} .
$$

We can consider $h_{a}$ as the sections $\Omega_{C^{\infty}}^{0}\left(\mathcal{U}_{a}, P\right)$ of the adjoint bundle $P=$ Aut $V$. We call the field $h$ a generalized tetrade field $(\mathrm{GTF})$. It follows from the definitions of the bases that there exists a global section for $e_{C^{\infty}}$

$$
e_{a}^{C^{\infty}}\left(z_{a}, \bar{z}_{a}\right)=e_{b}^{C^{\infty}}\left(z_{b}\left(z_{a}\right), \bar{z}_{b}\left(\bar{z}_{a}\right)\right), z_{a} \in \mathcal{U}_{a b}=\mathcal{U}_{a} \cap \mathcal{U}_{b} \neq \emptyset
$$

where $z_{b}=z_{b}\left(z_{a}\right)$ are holomorphic functions defining a complex structure on $\Sigma_{g}$. On the other hand the transformations of $e^{\text {hol }}$ are holomorphic maps

$$
\begin{gathered}
e_{a}^{h o l}\left(z_{a}\right)=g_{a b}\left(z_{a}\right) e_{b}^{h o l}\left(z_{b}\left(z_{a}\right)\right), \quad g_{b a}\left(z_{b}\right)=g_{a b}^{-1}\left(z_{a}\left(z_{b}\right)\right) \\
g_{a b} \in \Omega_{h o l}^{0}\left(\mathcal{U}_{a b}, \text { Aut } V\right), \quad\left(\bar{\partial} g_{a b}=0, \bar{\partial}=\partial_{\bar{z}_{a}}\right) .
\end{gathered}
$$

These matrix functions define the holomorphic structure in the vector bundle $V$.

We can describe the same holomorphic structure working with the smooth basis $e^{C^{\infty}}$ in $V$. Let

$$
\bar{A}_{a}=h_{a}^{-1} \bar{\partial} h_{a} .
$$

Then the basis $e^{C^{\infty}}$ is annihilated by the operator $\left.d_{A}^{\prime \prime}\right|_{\mathcal{U}_{a}}=\bar{\partial}+\bar{A}_{a}$

$$
\left(\bar{\partial}+\bar{A}_{a}\right) e_{a}^{C^{\infty}}=0 .
$$


The GTF transformations $h$ in (2.1) by no means free. Let $\mathcal{R}_{\Sigma}$ be the subset of sections in $P$ which satisfies the following conditions

$$
\begin{gathered}
\mathcal{R}_{\Sigma}=\left\{h \in \Omega_{C^{\infty}}^{0}\left(\mathcal{U}_{a}, P\right)\left|h_{a}^{-1} \bar{\partial} h_{a}\right|_{\mathcal{U}_{a b}}=\left.h_{b}^{-1} \bar{\partial} h_{b}\right|_{\mathcal{U}_{a b}}, \forall \mathcal{U}_{a b} \neq \emptyset, a, b=1, \ldots\right\} \\
\left(\bar{A}_{a}\left(z_{a}\right)=\bar{A}_{b}\left(z_{b}\left(z_{a}\right)\right), z_{a} \in \mathcal{U}_{a b}\right) .
\end{gathered}
$$

Proposition 2.1 Conditions (2.1) and (2.5) are equivalent.

Proof. Since $e_{b}^{C^{\infty}}=e_{a}^{C^{\infty}}$ in $\mathcal{U}_{a b}$ (2.1) implies

$$
g_{a b}=h_{a} h_{b}^{-1} .
$$

Then the holomorphicity of $g_{a b}$ implies (2.5) . If $h \in \mathcal{R}_{\Sigma}$, then (2.6) defines the transition map for some holomorphic basis $e^{h o l}$. The basis $e^{\text {hol }} h$ satisfies (2.2) and therefore can be taken as $e^{C^{\infty}}$.

Consider the group

$$
\mathcal{G}_{\Sigma}=\left\{\Omega_{C^{\infty}}^{0}\left(\mathcal{U}_{a}, P\right), a=1, \ldots\right\} .
$$

It transforms local basses of $V$ over $\mathcal{U}_{a}$. The group acts on itself by the left and right multiplications.

There are two subgroups of $\mathcal{G}_{\Sigma}$. Let $x_{a} \in \Omega_{C^{\infty}}^{0}\left(\mathcal{U}_{a}, P\right)$. Then

$$
\begin{gathered}
\mathcal{G}_{\Sigma}^{h o l}=\left\{x_{a} \rightarrow f_{a} x_{a} \mid f \in \Omega_{h o l}^{0}(\Sigma, P)\right\} \\
\mathcal{G}_{\Sigma}^{C^{\infty}}=\left\{x_{a} \rightarrow x_{a} \varphi_{a} \mid \varphi \in \Omega_{C^{\infty}}^{0}(\Sigma, P), \varphi\left(z_{b}\left(z_{a}\right), \bar{z}_{b}\left(\bar{z}_{a}\right)\right)=\varphi\left(z_{a}, \bar{z}_{a}\right) z_{a} \in \mathcal{U}_{a b}\right\} .
\end{gathered}
$$

We can consider the GTF (2.5) as a subset in $\mathcal{G}_{\Sigma}$. We have the following evident statement

Proposition 2.2 The left and right actions of $\mathcal{G}_{\Sigma}^{\text {hol }}$ and $\mathcal{G}_{\Sigma}^{C^{\infty}}$ leave invariant $\mathcal{R}_{\Sigma}$.

In other words

$$
\begin{array}{rccc}
\mathcal{G}_{\Sigma}: & \mathcal{G}_{\Sigma} & \stackrel{\text { right mltpl. }}{\longrightarrow} & \mathcal{G}_{\Sigma} \\
\cup & \cup & & \cup \\
\mathcal{G}_{\Sigma}^{C^{\infty}}: & \mathcal{R}_{\Sigma} & \stackrel{\text { right mltpl. }}{\longrightarrow} & \mathcal{R}_{\Sigma}
\end{array}
$$

and

$$
\begin{array}{cccc}
\mathcal{G}_{\Sigma}: & \mathcal{G}_{\Sigma} & \stackrel{\text { left mltpl. }}{\longrightarrow} & \mathcal{G}_{\Sigma} \\
\cup & \cup & & \cup \\
\mathcal{G}_{\Sigma}^{\text {hol }}: & \mathcal{R}_{\Sigma} & \stackrel{\text { left mltpl. }}{\longrightarrow} & \mathcal{R}_{\Sigma}
\end{array}
$$

2. Consider the space of holomorphic structures on the bundles $V$ and $P$. Since $g>1$ there is an open subset of stable holomorphic structures. The holomorphic structures can be defined in two ways. In the first type of the construction, which we call the 
D-type, the holomorphic structures are defined by the covariant operators. For $V$ they are

$$
d_{A}^{\prime \prime}: \Omega_{C^{\infty}}^{0}(\Sigma, V) \rightarrow \Omega_{C^{\infty}}^{0,1}(\Sigma, V) .
$$

It means that $\bar{A}$ satisfies (2.5). The holomorphic structure is consistent with the complex structure on $\Sigma_{g}$ such that for any section $s \in \Omega_{C^{\infty}}^{0}(\Sigma, V)$ and $f \in C^{\infty}(\Sigma) d_{A}^{\prime \prime}(f s)=$ $(\bar{\partial} f) s+f d_{A}^{\prime \prime} s$. The space of holomorphic structures $\mathcal{L}_{\Sigma}^{D}$ on $P$ is defined in the similar way

$$
\mathcal{L}_{\Sigma}^{D}=\left\{d_{A}^{\prime \prime}=\bar{\partial}+\bar{A}: \Omega_{C^{\infty}}^{0}(\Sigma, P) \rightarrow \Omega_{C^{\infty}}^{(0,1)}(\Sigma, P)\right\} .
$$

with the action in the adjoint representation. The stable holomorphic structures $\mathcal{L}_{\Sigma}^{D, s t}$ are an open subset in (2.10). The automorphisms of the holomorphic structures are given by the action of the gauge group $\mathcal{G}_{\Sigma}^{C^{\infty}}(2.9)$

$$
d_{A}^{\prime \prime} \rightarrow \varphi^{-1} d_{A}^{\prime \prime} \varphi, \varphi \in \mathcal{G}_{\Sigma}^{C^{\infty}}
$$

They preserve the subset $\mathcal{L}_{\Sigma}^{D, s t}$. The moduli space $\mathcal{L}$ of stable holomorphic structures on $P$ is the quotient space

$$
\mathcal{L}=\mathcal{L}_{\Sigma}^{D, s t} / \mathcal{G}_{\Sigma}^{C^{\infty}}
$$

It is a smooth complex manifold with tangent space at $\bar{A}$ is isomorphic to $H^{(0,1)}(\Sigma, \operatorname{Lie}(\mathrm{GL}(N, \mathbf{C})))$. Its dimension is given by the Riemann-Roch theorem

$$
\operatorname{dim} \mathcal{L}=N^{2}(g-1)+1 .
$$

The left action of the gauge transformations $\mathcal{G}_{\Sigma}^{\text {hol }}(2.8)$ does not change $\bar{A}_{a}=h_{a}^{-1} \bar{\partial} h_{a}, a=1, \ldots$. Therefore the space $\mathcal{L}_{\Sigma}^{D}(2.10)$ can be represented as the quotient space $\mathcal{L}_{\Sigma}^{D}=\mathcal{G}_{\Sigma}^{\text {hol }} \backslash \mathcal{R}_{\Sigma}$. There is an open subset in $\mathcal{R}_{\Sigma}^{\text {st }}$ such that the subset of the stable holomorphic structures is the quotient space

$$
\mathcal{L}_{\Sigma}^{D, s t}=\mathcal{G}_{\Sigma}^{\text {hol }} \backslash \mathcal{R}_{\Sigma}^{s t}
$$

The main statement of this section follows immediately from (2.12)

Proposition 2.3 The moduli space $\mathcal{L}$ of stable holomorphic structures on $P$ can be represented as the double coset space

$$
\mathcal{L}=\mathcal{G}_{\Sigma}^{\text {hol }} \backslash \mathcal{R}_{\Sigma}^{s t} / \mathcal{G}_{\Sigma}^{C^{\infty}}
$$

3. An alternative description of the holomorphic structures in terms of the Cech cohomologies, which we call the C-type construction is based on the transition maps (2.3), (2.6). The collection of transition maps

$$
\mathcal{L}_{\Sigma}^{C h}=\left\{g_{a b}\left(z_{a}\right)=h_{a}\left(z_{a}\right) h_{b}^{-1}\left(z_{b}\left(z_{a}\right)\right), z_{a} \in \mathcal{U}_{a b}, a, b=1, \ldots,\right\} .
$$

defines the holomorphic structures on $V$ or $P$ depending on the choice of the representations. Again we choose the open subset of stable holomorphic structures $\mathcal{L}_{\Sigma}^{C, s t}$ in $\mathcal{L}_{\Sigma}^{C h}$. The gauge group $\mathcal{G}_{\Sigma}^{\text {hol }}$ acts as the automorphisms of $\mathcal{L}_{\Sigma}^{C, s t}$

$$
g_{a b} \rightarrow f_{a} g_{a b} f_{b}^{-1}, f_{a}=f\left(z_{a}\right), f_{b}=f_{b}\left(z_{b}\left(z_{a}\right)\right), f \in \mathcal{G}_{\Sigma}^{h o l} .
$$


The space $\mathcal{L}_{\Sigma}^{C h}$ has a transparent description in terms of graphs. Consider the skeleton of the covering $\left\{\mathcal{U}_{a}, a=1, \ldots\right\}$. It is an oriented graph, whose vertices are some fixed inner points in $\mathcal{U}_{a}$ and edges $L_{a b}$ connect those $V_{a}$ and $V_{b}$ for which $U_{a b} \neq \emptyset$. We choose an orientation of the graph, saying that $a>b$ on the edge $L_{a b}$ and put the holomorphic function $z_{b}\left(z_{a}\right)$ which defines the holomorphic map from $\mathcal{U}_{a}$ to $\mathcal{U}_{b}$. Then the space $\mathcal{L}_{\Sigma}^{C h}$ can be defined by the following data. To each edge $L_{a b}, a>b$ we attach a matrix valued function $g_{a b} \in \operatorname{GL}(N, \mathbf{C})$ along with $z_{b}\left(z_{a}\right)$. The gauge fields $f_{a}$ are living on the vertices $V_{a}$ and the gauge transformation is (2.16).

The moduli space of stable holomorphic bundles is defined as the factor space under this action

$$
\mathcal{L}=\mathcal{G}_{\Sigma}^{\text {hol }} \backslash \mathcal{L}_{\Sigma}^{C h, s t}
$$

The tangent space to the moduli space in this approach is $H^{1}(\Sigma, \operatorname{Lie}(\mathrm{GL}(N, \mathbf{C})))$ extracted from the Cech complex. Though $\mathcal{L}_{\Sigma}^{C h, s t}$ differs from $\mathcal{L}_{\Sigma}^{D, s t}$ we obtain the same moduli space $\mathcal{L}$ of stable holomorphic structures on $P$ due to the equivalence of the Dolbeault and the Cech cohomologies.

In this construction the right action of $\mathcal{G}_{\Sigma}^{C^{\infty}}$ (2.9) leaves the transition maps $g_{a b}$ invariant. Therefore

$$
\mathcal{L}_{\tilde{\Sigma}}^{C h, s t}=\mathcal{R}_{\Sigma}^{s t} / \mathcal{G}_{\Sigma}^{C^{\infty}} .
$$

Taking into account (2.17) we come to the same construction of the moduli space as the double coset space (2.14).

4. We fit the components of our construction in the exact bicomplex

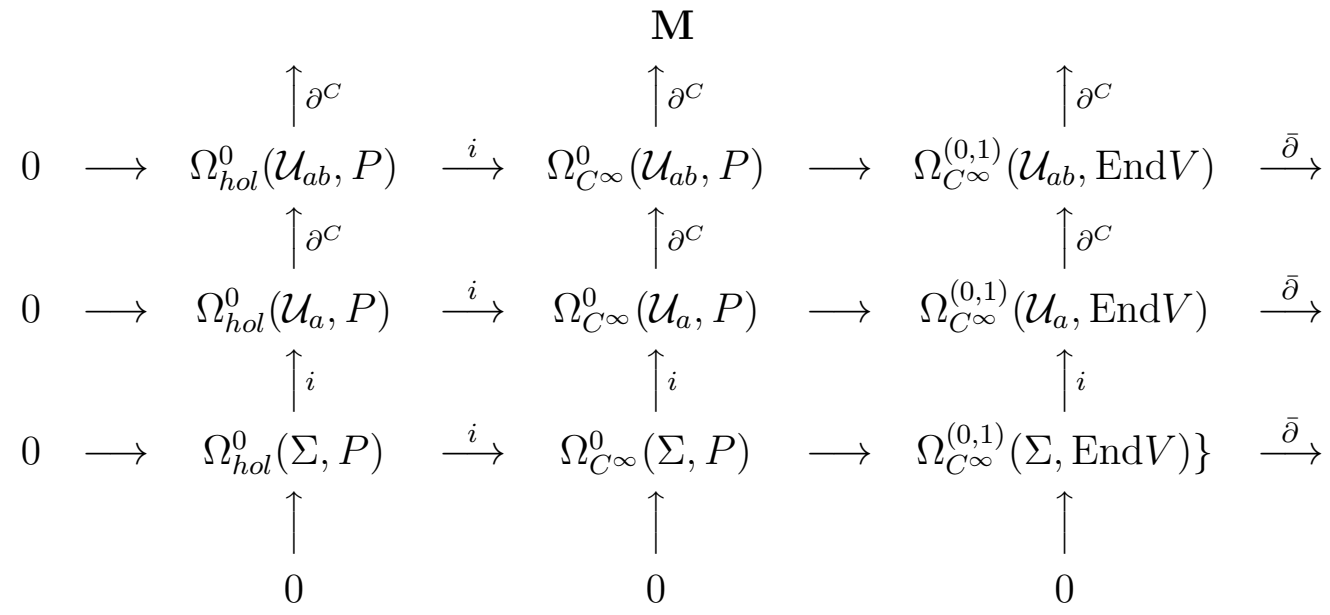

Here $\partial^{C}$ are the Ĉch differentials, $i$ are the augmentations. The right arrows from $\Omega_{C^{\infty}}^{0}(*, P)$ to $\Omega_{C^{\infty}}^{(0,1)}(*, \operatorname{End} V)$ are of the type $h \rightarrow h^{-1} \bar{\partial} h$. We have

$$
\begin{gathered}
g_{a b} \in \Omega_{C^{\infty}}^{(0,1)}\left(\mathcal{U}_{a b}, \operatorname{End} V\right), h_{a} \in \Omega_{C^{\infty}}^{0}\left(\mathcal{U}_{a}, P\right), \\
\delta \bar{A} \in \Omega_{C^{\infty}}^{(0,1)}\left(\mathcal{U}_{a}, \text { End } V\right) .
\end{gathered}
$$

If these fields satisfy the tetrade conditions $(2.1),(2.3)$ or $(2.5)$ then they lie in the images of $i$. The Dolbeault cohomologies $H^{(0,1)}(\Sigma$, End $V)$ that define the tangent space to the moduli space are living in $\Omega_{C^{\infty}}^{(0,1)}(\Sigma$, End $V)$ and the Cech cohomologies $H^{1}(\Sigma$, End $V)$ in 
$\Omega_{h o l}^{0}\left(\mathcal{U}_{a b}\right.$, End $\left.V\right)$. Their equivalence can be derived from the properties of the double spectral sequence.

The gauge transformations also can be incorporated in the exact bicomplex

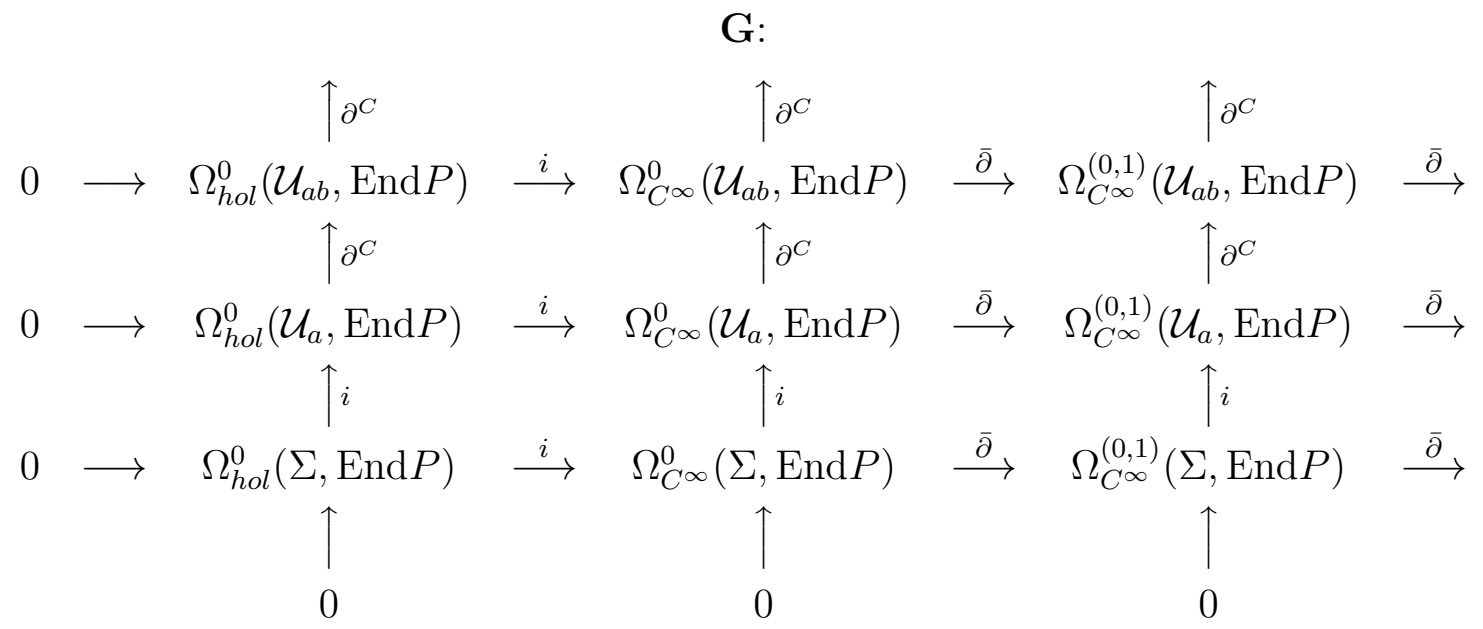

Let $\epsilon^{\text {hol }} \in \operatorname{Lie}\left(\mathcal{G}_{\Sigma}^{h o l}\right), \epsilon^{C^{\infty}} \in \operatorname{Lie}\left(\mathcal{G}_{\Sigma}^{C^{\infty}}\right)$. Then

$$
\begin{gathered}
\epsilon^{\text {hol }} \in \text { Image of }\left(\Omega_{h o l}^{0}\left(\mathcal{U}_{a}, \operatorname{End} P\right)\right) \text { in } \Omega_{C^{\infty}}^{0}\left(\mathcal{U}_{a}, \operatorname{End} P\right), \\
\epsilon^{C^{\infty}} \in \text { Image of }\left(\Omega_{C^{\infty}}^{0}(\Sigma, \text { End } P) \text { in } \Omega_{C^{\infty}}^{0}\left(\mathcal{U}_{a}, \operatorname{End} P\right) .\right.
\end{gathered}
$$

The actions of the gauge group (see (2.11) and (2.16))

$$
\begin{gathered}
\delta^{C^{\infty} \bar{A}_{a}}=\bar{\partial} \epsilon_{a}^{C^{\infty}}+\left[\bar{A}_{a}, \epsilon_{a}^{C^{\infty}}\right], \\
\delta^{h o l} g_{a b}=\epsilon_{a}^{h o l} g_{a b}-g_{a b} \epsilon_{b}^{h o l} .
\end{gathered}
$$

More generally, $\mathbf{M}$ is the bigraded $\mathbf{G}$ module. The action of $\mathbf{G}$ is consistent with the both differential $\partial^{C}$ and $\bar{\partial}$. The differentiations take into account the bigradings of $\mathbf{M}$ and $\mathbf{G}$. The actions (2.19), (2.20) are particular cases of these actions .

\section{The Schottky specialization.}

We apply the general scheme to the particular covering of $\Sigma_{g}$ based on the Schottky parameterization. Consider the Riemann sphere with $2 g$ circles $\mathcal{A}_{a}, \mathcal{A}_{a}^{\prime}, a=1, \ldots g$. Each circle lies in the external part of others. Let $\gamma_{a}$ be $g$ projective maps $\mathcal{A}_{a}^{\prime}=\gamma_{a} \mathcal{A}_{a}, \gamma_{a} \in$ $P S L(2)$. The Schottky group $\Gamma$ is a free group generated by $\gamma_{a}, a=1 \ldots g$. The exterior part of all the circles

$$
\tilde{\Sigma}=\mathbf{P}^{1} / \cup_{b=1}^{2 g} D_{b}
$$

is the fundamental domain of $\Gamma$. The surface $\Sigma$ is obtained from $\tilde{\Sigma}$ by the pairwise gluing of the circles $\mathcal{A}_{a}^{\prime}=\gamma_{a} \mathcal{A}_{a}$ and $\mathcal{A}_{a}$. We have only one nonsimpliconnected $2 \mathrm{~d}$ cell $\mathcal{U}_{a} \sim \tilde{\Sigma}$ with selfintersections $\mathcal{U}_{a a^{\prime}}=$ vicinity $\mathcal{A}_{a}=$ vicinity $\mathcal{A}_{a}^{\prime}$. We choose $g$ local coordinates $z_{a}, a=1, \ldots, g$, which define the parameterizations of the internal disks of $\mathcal{A}_{a}$ circles. In 
this case the holomorphic maps can be written as $z_{a^{\prime}}\left(z_{a}\right)=\gamma_{a}\left(z_{a}\right)$. The GTF $\mathcal{R}_{\Sigma}(2.5)$ is a twisted field $h$ on $\tilde{\Sigma}$

$$
h^{-1} \bar{\partial} h\left(z_{a}, \bar{z}_{a}\right)=h^{-1} \bar{\partial} h\left(\gamma_{a}\left(z_{a}\right), \overline{\gamma_{a}\left(z_{a}\right)}\right), a=1, \ldots, g .
$$

In the definition of $\mathcal{G}_{\Sigma}^{C^{\infty}}$ (2.9) "the periodicity conditions" take the form

$$
\varphi\left(\gamma_{a}\left(z_{a}\right), \bar{\gamma}_{a}\left(\bar{z}_{a}\right)\right)=\varphi\left(z_{a}, \bar{z}_{a}\right), z_{a} \in \text { vicinity of } \mathcal{A}_{a} .
$$

The transition maps (2.3), 2.6) defining $\mathcal{L}_{\Sigma}^{C h}$ are

$$
g_{a}=g_{a a^{\prime}}\left(z_{a}\right)=h\left(z_{a}, \bar{z}_{a}\right) h^{-1}\left(\gamma_{a}\left(z_{a}\right), \bar{\gamma}_{a}\left(\bar{z}_{a}\right)\right), a=1, \ldots, g .
$$

The gauge group $\mathcal{G}_{\Sigma}^{\text {hol }}$ acts as a global holomorphic transformation on $\tilde{\Sigma}$. In the local coordinates we have

$$
g_{a} \rightarrow f_{a} g_{a} f_{\gamma_{a}}^{-1}, f_{a}=f\left(z_{a}\right), g_{a}=g_{a}\left(z_{a}\right), f_{\gamma_{a}}=f\left(\gamma_{a}\left(z_{a}\right)\right) .
$$

In local coordinates $g_{a}$ have the form of Laurent polynomials. $g_{a}\left(z_{a}\right)=\sum g_{a}^{(k)} z_{a}^{k}$. Thus in this parameterization the set of holomorphic structures on the vector bundles $\mathcal{L}_{\Sigma}^{C h}$ can be identified with the collection of the loop groups $L\left(G L_{a}(N, \mathbf{C})\right)$. But in fact, taking into account the adjoint action of the gauge group (3.2), one concludes that the precise form of components is the semidirect product $L(\mathrm{GL}(N, \mathbf{C}))>\triangleleft P S L(2)=\{g(z)>\triangleleft \gamma(z)\}$. Thus

$$
\mathcal{L}_{\Sigma}^{C h}=\oplus_{a=1}^{g} L_{a}(\mathrm{GL}(N, \mathbf{C}))>\triangleleft P S L(2)_{a},
$$

where the subgroups $\left\{P S L(2)_{a}\right\}, a=1 \ldots g$ are responsible for the complex structure on $\Sigma$. To define the stable bundles one should choose an open subset in $L_{a}(G L(N, \mathbf{C}))$.

Consider the bundles over genus $g=1$ curves. Though the bundles are unstable this case can be completely described in the wellknown terms. The Schottky parameterization means the realization of elliptic curve as an annulus. Let $\gamma(z)=q z, q=\exp (2 \pi i \tau)$. The holomorphic bundles are defined by the loop group extended by the shift operator

$$
\mathcal{L}_{\Sigma}^{C h}=L(G L(N, \mathbf{C}))>\triangleleft \exp (2 \pi i \tau z \partial) .
$$

The gauge action (3.2)

$$
g(z) \rightarrow f(z) g(z) f^{-1}(q z)
$$

transforms $g(z)$ to a $z$ independent diagonal form, up to the action of the complex affine Weyl group $\hat{W}$. Let $W$ be the $A_{N-1}$ Weyl group (the permutations of the Cartan elements). Then $\hat{W}=\left(\mathbf{Z} R^{\vee} \tau+\mathbf{Z} R^{\vee}\right)>\triangleleft W\left(R^{\vee}\right.$ is the dual root system). The moduli space $\mathcal{L}$ in this case is the Weyl alcove. The comparison of two description of holomorphic structures on elliptic curves (3.4) and (2.10) was carried out in [11, 12 in terms of two loop current algebras and invariants of their coadjoint actions.

In general case $(g>1)$ the gauge transform (3.2) allows to choose $g_{a}$ as constant matrices. They are defined up to the common conjugation by a $\operatorname{GL}(N, \mathbf{C})$ matrix. Thus the moduli space of holomorphic bundles in the (3.3) description are defined as the quotient

$$
\mathcal{L} \sim(\underbrace{\mathrm{GL}(N, \mathbf{C}) \oplus \ldots \oplus \mathrm{GL}(N, \mathbf{C})}_{g}) / \mathrm{GL}(N, \mathbf{C}) .
$$

Since the center of $\operatorname{GL}(N, \mathbf{C})$ acts trivially we obtain $\operatorname{dim} \mathcal{L}=N^{2}(g-1)+1($ see $(2.13))$. 


\section{Symplectic geometry in the double coset picture}

Here we consider the Hitchin integrable systems which are defined on the cotangent bundle $T^{*} \mathcal{L}$ to the moduli of stable holomorphic bundles $\mathcal{L}$. As it was done in the original work [7] this space is derived as a symplectic quotient of $T^{*} \mathcal{L}_{\Sigma}^{D}$ under the gauge action of $\mathcal{G}_{\Sigma}^{C^{\infty}}$. We will come to the same systems by the three step symplectic reductions from some big upstairs space. The main object of this section is the commutative diagram (4.10), which describes these reductions and intermediate spaces.

1. First, as intermediate step, consider the Hitchin description of $T^{*} \mathcal{L}$. The upstairs phase space is the cotangent bundle $T^{*} \mathcal{L}_{\Sigma}^{D}$ to the space $\mathcal{L}_{\Sigma}^{D}(2.10)$ of holomorphic structures on the bundle $P$ in the Dolbeault picture. It is the space of pairs

$$
T^{*} \mathcal{L}_{\Sigma}^{D}=\left\{\phi, d_{A}^{\prime \prime}, \quad \phi \in \Omega_{C^{\infty}}^{(1,0)}\left(\Sigma,(\operatorname{End} V)^{*}\right)\right\}
$$

The field $\phi$ is called the Higgs field and the bundle $T^{*} \mathcal{L}_{\Sigma}^{D}$ is the Higgs bundle. We can consider the Higgs field as a form

$$
\phi \in \Omega_{C^{\infty}}^{0}\left(\Sigma,(\operatorname{End} V)^{*} \otimes K\right),
$$

where $K$ is the canonical bundle on $\Sigma$. Locally on $\mathcal{U}_{a}$

$$
d_{a}^{\prime \prime}=\bar{\partial}+\bar{A}_{a}, \bar{A}_{a}=h_{a}^{-1} \bar{\partial} h_{a}, h_{a} \in \Omega_{C^{\infty}}^{0}\left(\mathcal{U}_{a}, \mathcal{R}_{\Sigma}\right)
$$

The symplectic form on it is

$$
\omega^{D}=\int_{\Sigma} \operatorname{tr}\left(D \phi, D d_{A}^{\prime \prime}\right)
$$

The action of the gauge group $\mathcal{G}_{\Sigma}^{C^{\infty}}$ (2.9) on $d_{A}^{\prime \prime}$ (2.11) with

$$
\phi \rightarrow \varphi^{-1} \phi \varphi
$$

is a symmetry of $T^{*} \mathcal{L}_{\Sigma}^{D}$. It defines the moment map

$$
\begin{gathered}
\mu_{\mathcal{G}_{\Sigma}^{C}}(\phi, \bar{A}): T^{*} \mathcal{L}_{\Sigma}^{D} \rightarrow \operatorname{Lie}^{*}\left(\mathcal{G}_{\Sigma}^{C^{\infty}}\right), \\
\mu_{\mathcal{G}_{\Sigma}^{C}}(\phi, \bar{A})=\left[d_{A}^{\prime \prime}, \phi\right] .
\end{gathered}
$$

For the zero level moment map $\left[d_{A}^{\prime \prime}, \phi\right]=0$ the Higgs field becomes holomorphic

$$
\phi \in H^{0}\left(\Sigma,(\operatorname{End} V)^{*} \otimes K\right) .
$$

The symplectic quotient $\mu^{-1}(0) / \mathcal{G}_{\Sigma}^{C^{\infty}}=T^{*} \mathcal{L}_{\Sigma}^{D} / / \mathcal{G}_{\Sigma}^{C^{\infty}}$ is identified with the cotangent bundle to the moduli space $T^{*} \mathcal{L}$. The Hitchin commuting integrals are constructed by means of $(1-j, 1)$ holomorphic differentials $\nu_{j, k}, k=1, \ldots$ :

$$
I_{j, k}^{D}=\int_{\Sigma} \nu_{j, k}^{D} \operatorname{tr} \phi^{j}
$$

Since the space of these differentials $H^{0}\left(\Sigma, K \otimes T^{j}\right)$ ( $K$ is the canonical class, $T^{j}$ is $(-j, 0)$ forms $)$ has dimension $(2 j-1)(g-1)$ for $j>1$ and $g$ for $j=1$ we have 
$N^{2}(g-1)+1$ independent commuting integrals, providing the complete integrability of the Hamiltonian systems (4.2), (4.3). The integrals (4.3) define the Hitchin map

$$
H^{0}\left(\Sigma,(\operatorname{End} V)^{*} \otimes K\right) \rightarrow H^{0}\left(\Sigma, K^{j}\right)
$$

2. The same system can be derived starting from the cotangent bundle $T^{*} \mathcal{L}_{\Sigma}^{C h}$ to the holomorphic structures on $P$ defined in the C-type description (2.15). Now

$$
T^{*} \mathcal{L}_{\Sigma}^{C h}=\left\{\eta_{a b}, g_{a b} \mid \eta_{a b} \in \Omega_{h o l}^{(1,0)}\left(\mathcal{U}_{a b},(\text { End } V)^{*}\right), g_{a b} \in \Omega_{h o l}^{0}\left(\mathcal{U}_{a b}, P\right)\right\}
$$

This bundle can be endowed with the symplectic structure by means of the Cartan-Maurer one-forms on $\Omega_{h o l}^{0}\left(\mathcal{U}_{a b}, P\right)$. Let $\Gamma_{a}^{b}(C, D)$ be a path in $\mathcal{U}_{a b}$ with the end points in the triple intersections $C \in \mathcal{U}_{a b c}=\mathcal{U}_{a} \cap \mathcal{U}_{b} \cap \mathcal{U}_{c}, D \in \mathcal{U}_{a b d}$. We can put the data (4.4) on the fat graph corresponding to the covering $\left\{\mathcal{U}_{a}\right\}$. The edges of the graph are $\left\{\Gamma_{a}^{b}(C D)\right\}$ and $\left\{\Gamma_{b}^{a}(D C)\right\}$ with opposite orientation. We assume that the covering is such that the orientation of edges defines the oriented contours around the faces $\mathcal{U}_{a}$. The fields $\eta_{a b}, g_{a b}$ are attributed to the edge $\Gamma_{a}^{b}(C D)$, while $\eta_{b a}, g_{b a}$ to $\Gamma_{b}^{a}(D C)$. The last pair is not independent $-\left(g_{a b}^{-1}=g_{b a}\right)$ (see (2.3)). Its counterpart in the dual space is

$$
\eta_{a b}\left(z_{a}\right)=g_{a b}\left(z_{a}\right) \eta_{b a}\left(z_{b}\left(z_{a}\right)\right) g_{a b}^{-1}\left(z_{a}\right)
$$

The symplectic structure is defined by the form

$$
\omega^{C h}=\sum_{\text {edges }} \int_{\Gamma_{a}^{b}(C D)} D \operatorname{tr}\left(\eta_{a b}\left(z_{a}\right)\left(D g_{a b} g_{a b}^{-1}\right)\left(z_{a}\right)\right) .
$$

Here the sum is taken over the edges of the oriented graph obtained from the fat graph after the identifications of fields (4.5). In other words we consider only the edge $\Gamma_{a}^{b}$ with the fields $g_{a b}, \eta_{a b}$ and forget about the edge $\Gamma_{b}^{a}$. Since $\eta_{a b}$ and $g_{a b}$ are holomorphic in $\mathcal{U}_{a b}$, the definition is independent on a choice of the path $\Gamma_{a}^{b}$ within $\mathcal{U}_{a b}$. The symplectic form is invariant under the gauge transformations (2.16) supplemented by

$$
\eta_{a b} \rightarrow f_{a} \eta_{a b} f_{a}^{-1}
$$

The set of invariant commuting integrals on $T^{*} \mathcal{L}_{\Sigma}^{C h}$ is

$$
I_{j, k}^{C h}=\sum_{\text {edges }} \int_{\Gamma_{a}^{b}(C D)} \nu_{(j, k)}^{C h}\left(z_{a}\right) \operatorname{tr}\left(\eta_{a b}^{j}\left(z_{a}\right)\right),
$$

where $\nu_{j, k}^{C h}$ are $(1-j, 0)$ differentials, which are related locally to the $(1-j, 1)$ differentials as $\nu_{j, k}^{D}=\bar{\partial} \nu_{j, k}^{C h}$.

We can consider the system on the defined above graph $L_{a b}$ which is dual to $\Gamma_{a}^{b}(C D)$. The fields $g_{a b}, \eta_{a b}, a, b=1 \ldots$ are defined on edges, while the gauge transformations $f_{a}$ live on vertices.

The moment map is

$$
\mu_{\mathcal{G}_{\Sigma}^{\text {hol }}}\left(\eta_{a b}, g_{a b}\right): T^{*} \mathcal{L}_{\Sigma}^{C h} \rightarrow \operatorname{Lie}^{*}\left(\mathcal{G}_{\Sigma}^{h o l}\right)
$$


According to (2.20) the Hamiltonian generating the gauge transformations is

$$
\begin{gathered}
F_{\epsilon^{h o l}}=\sum_{\text {edges }} \int_{\Gamma_{a}^{b}(C D)} \operatorname{tr}\left(\eta_{a b}\left(z_{a}\right) \epsilon_{a}^{h o l}\left(z_{a}\right)\right)-\operatorname{tr}\left(\eta_{a b}\left(z_{a}\right) g_{a b}\left(z_{a}\right) \epsilon_{b}^{h o l}\left(\left(z_{b}\left(z_{a}\right)\right) g_{a b}\left(z_{a}\right)^{-1}\right)=\right. \\
\sum_{\text {edges }} \int_{\Gamma_{a}^{b}(C D)} \operatorname{tr}\left(\eta_{a b}\left(z_{a}\right) \epsilon_{a}^{h o l}\left(z_{a}\right)\right)-\operatorname{tr}\left(\eta_{b a}\left(z_{b}\left(z_{a}\right)\right) \epsilon_{b}^{h o l}\left(z_{b}\left(z_{a}\right)\right)\right)= \\
\sum_{a} \int_{\Gamma_{a}} \sum_{b} \operatorname{tr}\left(\eta_{a b}\left(z_{a}\right) \epsilon_{a}^{h o l}\left(z_{a}\right)\right),
\end{gathered}
$$

where $\Gamma_{a}$ is an oriented contour around $\mathcal{U}_{a}$. The moment equation $\mu_{\mathcal{G}_{\Sigma}^{\text {hol }}}=0$ can be read off from $F_{\epsilon}$ hol. It means that $\eta_{a b}$ is a boundary value of some holomorpfic form defined on $\mathcal{U}_{a}$

$$
\eta_{a b}\left(z_{a}\right)=H_{a}\left(z_{a}\right), \text { for } z_{a} \in \mathcal{U}_{a b}, H_{a} \in \Omega_{h o l}^{(1,0)}\left(\mathcal{U}_{a},(\operatorname{End} V)^{*}\right) .
$$

The reduced system is again the cotangent bundle to the moduli space of holomorphic bundles

$$
T^{*} \mathcal{L}=\mathcal{G}_{\Sigma}^{\text {hol }} \backslash \backslash T^{*} \mathcal{L}_{\Sigma}^{C h}=\mathcal{G}_{\Sigma}^{\text {hol }} \backslash \mu_{\mathcal{G}_{\Sigma}^{\text {hol }}}^{-1}(0),
$$

which has dimension $2 N^{2}(g-1)+2$.

3. To get the cotangent bundle $T^{*} \mathcal{L}$ via the symplectic reduction we can start from $T^{*} \mathcal{R}_{\Sigma}$ using the double coset representation (2.14). Then $T^{*} \mathcal{L}_{\Sigma}^{D}$ or $T^{*} \mathcal{L}_{\Sigma}^{C h}$ are obtained on the intermediate stages of the two step reduction under the actions of $\mathcal{G}_{\Sigma}^{\text {hol }}$ or $\mathcal{G}_{\Sigma}^{C^{\infty}}$. Since these groups act from different sides on $\mathcal{R}_{\Sigma}$ their actions commute and the result of the reduction procedure is independent on their order. But the space $\mathcal{R}_{\Sigma}$, as we already have remarked, is not free - its elements satisfy (2.5). We will represent the constraints (2.5) as moment constraints and consider the "superfree" space - cotangent bundle to the group $\mathcal{G}_{\Sigma}$ (2.7). More exactly we will consider (Theorem 4.1) the three step symplectic reductions which result in the following commutative "tadpole" diagram

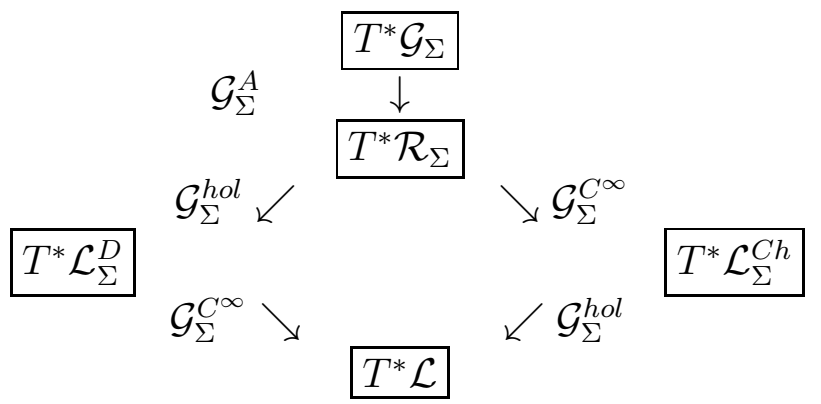

To begin with we define the initial data on $T^{*} \mathcal{G}_{\Sigma}$ and the gauge group $\mathcal{G}_{\Sigma}^{A}$. To construct $T^{*} \mathcal{G}_{\Sigma}$ we consider three dual elements

$$
\begin{gathered}
\Psi_{a} \in \Omega_{C^{\infty}}^{(1,1)}\left(\mathcal{U}_{a},(\operatorname{End} V)^{*}\right), \eta_{a b}, \eta_{b a} \in \Omega_{C^{\infty}}^{(1,0)}\left(\mathcal{U}_{a b},(\operatorname{End} V)^{*}\right), \\
\xi_{a b}, \xi_{b a} \in \Omega_{C^{\infty}}^{(0,1)}\left(\mathcal{U}_{a b},(\operatorname{End} V)^{*}\right) .
\end{gathered}
$$


Cotangent bundle $T^{*} \mathcal{G}_{\Sigma}$ is the set of fields $(\Psi, \eta, \xi, h)$. We endow it with the symplectic structure. Consider the same fat graph with edges $\Gamma_{a}^{b}(C D)$ and $\Gamma_{b}^{a}(D C)$ as in 4.2. Then

$$
\omega_{\Sigma}=D\left\{\sum_{a} \int_{\mathcal{U}_{a}} \operatorname{tr}\left(\Psi_{a} D h_{a} h_{a}^{-1}\right)+\sum_{b}\left[\int_{\Gamma_{a}^{b}} \operatorname{tr}\left(\eta_{a b} D h_{a} h_{a}^{-1}\right)+\int_{\Gamma_{a}^{b}} \operatorname{tr}\left(\xi_{a b} h_{a}^{-1} D h_{a}\right)\right]\right\} .
$$

We assume as before that paths $\Gamma_{a}^{b}, \Gamma_{a}^{c}, \ldots$ can be unified in a closed oriented contour $\Gamma_{a} \subset \mathcal{U}_{a}$. The integral over $\mathcal{U}_{a}$ means in fact the integral over a part of $\mathcal{U}_{a}$ restricted by the contour $\Gamma_{a}$. Thus the first sum can be replaced by the integration over $\Sigma$. To maintain the independence of $\omega_{\Sigma}$ on the choice of the contours $\Gamma_{a}$ we introduce the following "gauge" symmetry. Its action defines of variations of fields along with variations of contours. Let $\Gamma_{a}^{\prime}$ be another contour and $\delta \mathcal{U}_{a}$ be the corresponding variation of the integration domain. There is the integral relation between fields coming from the Stokes theorem, providing the independence of $\omega_{\Sigma}$

$$
\begin{gathered}
\int_{\delta \mathcal{U}_{a}} \operatorname{tr}\left(\Psi_{a} D h_{a} h_{a}^{-1}\right)=\left[\int_{\Gamma_{a}^{\prime}} \operatorname{tr}\left(\eta_{a b} D h_{a} h_{a}^{-1}\right)+\int_{\Gamma_{a}^{\prime}} \operatorname{tr}\left(\xi_{a b} h_{a}^{-1} D h_{a}\right)\right] \\
-\left[\int_{\Gamma_{a}} \operatorname{tr}\left(\eta_{a b} D h_{a} h_{a}^{-1}\right)+\int_{\Gamma_{a}} \operatorname{tr}\left(\xi_{a b} h_{a}^{-1} D h_{a}\right)\right] .
\end{gathered}
$$

In other words, the variation of contours is compensated by the variation of the field $\Psi$.

The form $\omega_{\Sigma}$ (4.11) is invariant under the actions of $\mathcal{G}_{\Sigma}^{\text {hol }}$ :

$$
h_{a} \rightarrow f_{a} h_{a}, \Psi_{a} \rightarrow f_{a} \Psi_{a} f_{a}^{-1}, \eta_{a b} \rightarrow f_{a} \eta_{a b} f_{a}^{-1}, \xi_{a b} \rightarrow \xi_{a b}
$$

and $\mathcal{G}_{\Sigma}^{C^{\infty}}$

$$
h_{a} \rightarrow h_{a} \varphi_{a}, \Psi_{a} \rightarrow \Psi_{a}, \eta_{a b} \rightarrow \eta_{a b}, \xi_{a b} \rightarrow \varphi_{a}^{-1} \xi_{a b} \varphi_{a}
$$

We extend these group transformations by the following affine action of the group

$$
\mathcal{G}_{\Sigma}^{A}=\left\{s_{a b} \in \Omega_{C^{\infty}}^{0}\left(\mathcal{U}_{a b}, P\right) \mid s_{a b}=s_{b a}\right\}
$$

on $\xi_{a b}$

$$
\xi_{a b} \rightarrow \xi_{a b}-s_{a b}^{-1}\left(\bar{\partial}+h_{a}^{-1} \bar{\partial} h_{a}\right) s_{a b}+h_{a}^{-1} \bar{\partial} h_{a}
$$

leaving the other fields untouched. This action commutes with $\mathcal{G}_{\Sigma}^{\text {hol }}$, but does not commute with $\mathcal{G}_{\Sigma}^{C^{\infty}} \cdot \mathcal{G}_{\Sigma}^{A}$ can be imbedded in the bicomplex $\mathbf{G}$ (see (4.15)). On the Lie algebra level we have

$$
\begin{gathered}
\xi_{a b} \rightarrow \xi_{a b}-\left(\bar{\partial} \epsilon_{a b}^{A}+\left[h_{a}^{-1} \bar{\partial} h_{a}, \epsilon_{a b}^{A}\right]\right) \\
\left(\epsilon_{a b}^{A} \in \operatorname{Lie}\left(\mathcal{G}_{\Sigma}^{A}\right)=\left\{\Omega_{C^{\infty}}^{0}\left(\mathcal{U}_{a b}, \text { End } V\right) \mid \epsilon_{a b}^{A}=\epsilon_{b a}^{A}\right\} .\right.
\end{gathered}
$$

Proposition 4.1 The form $\omega_{\Sigma}\left(\sqrt{4.11}\right.$ ) is invariant under the action of $\mathcal{G}_{\Sigma}^{A}$. 
Proof. From (4.16)

$$
\delta_{\epsilon_{a b}^{A}} \xi_{a b}=-\left(\bar{\partial} \epsilon_{a b}^{A}+\left[h_{a}^{-1} \bar{\partial} h_{a}, \epsilon_{a b}^{A}\right]\right),
$$

where $\epsilon^{A} \in \operatorname{Lie}\left(\mathcal{G}_{\Sigma}^{A}\right)$. Then

$$
\begin{gathered}
-\delta_{\epsilon^{A}} \omega_{\tilde{\Sigma}}=\sum_{a} \sum_{b} \int_{\Gamma_{a}^{b}} \operatorname{tr}\left\{D\left(\left[h_{a}^{-1} \bar{\partial} h_{a}, \epsilon_{a b}^{A}\right]\right) h_{a}^{-1} D h_{a}+\bar{\partial} \epsilon_{a}^{A} D\left(h_{a}^{-1} D h_{a}\right)+\left[h_{a}^{-1} \bar{\partial} h_{a}, \epsilon_{a}^{A}\right] D\left(h_{a}^{-1} D h_{a}\right)\right\} \\
=-\sum_{a} \sum_{b} \int_{\Gamma_{a}^{b}} \operatorname{tr}\left\{\left[D\left(h_{a}^{-1} D h_{a}\right), h_{a}^{-1} D h_{a}\right]+\bar{\partial} D\left(h_{a}^{-1} D h_{a}\right)+\left[h_{a}^{-1} \bar{\partial} h_{a}, D\left(h_{a}^{-1} D h_{a}\right)\right], \epsilon_{a b}^{A}\right\} .
\end{gathered}
$$

Then direct calculations show that the sum under the integral in front of $\epsilon_{a b}^{A}$ vanishes. Therefore $\omega_{\Sigma}$ is invariant under these transformations.

More generally, the dual fields $\left(\Psi_{a}, \eta_{a b}, \eta_{b a}, \xi_{a b}, \xi_{b a}\right)$ can be incorporated in a general pattern of two exact $\mathbf{G}$ bimoduli:
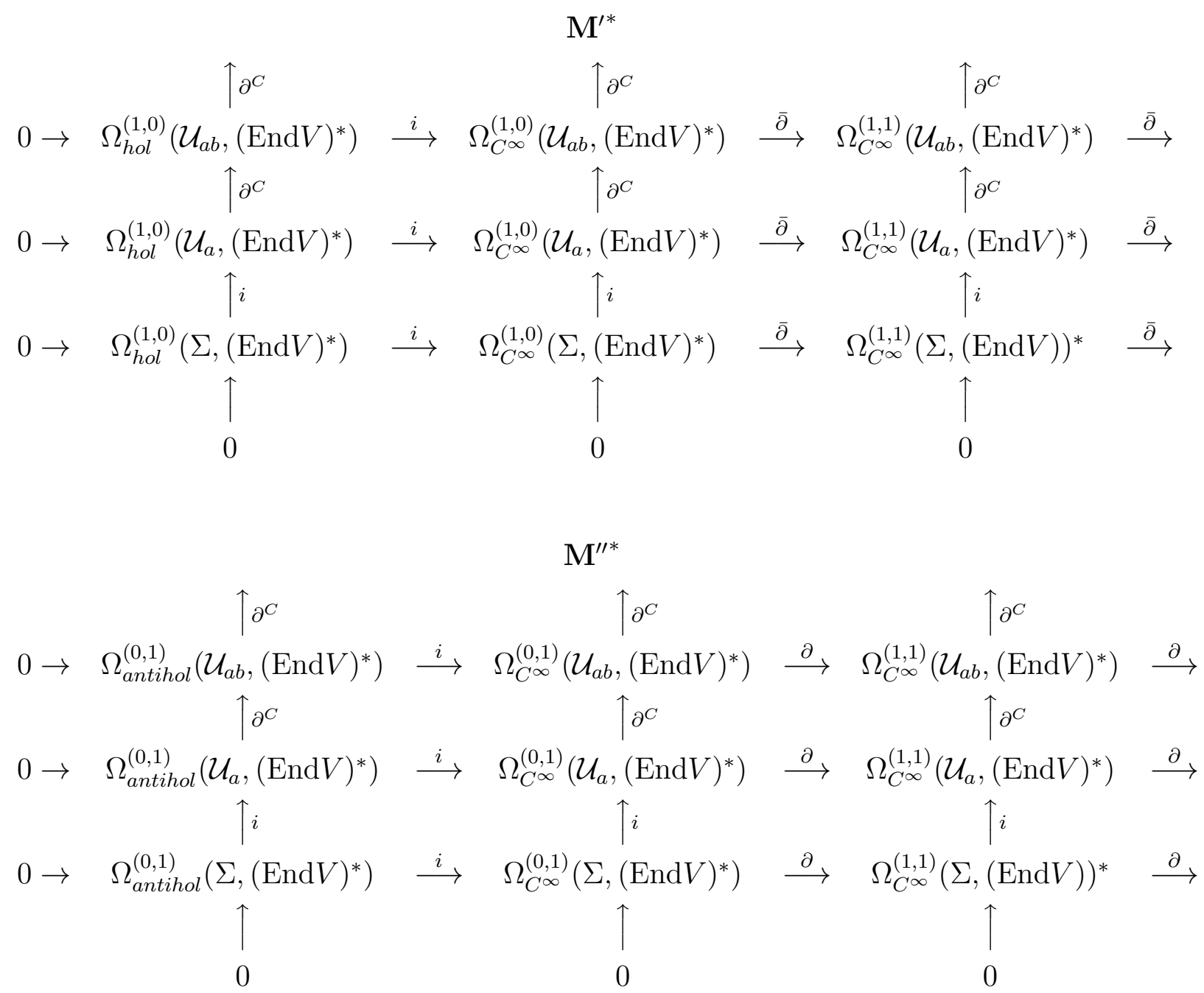

We remind that

$$
\Psi_{a} \in \Omega_{C^{\infty}}^{(1,1)}\left(\mathcal{U}_{a},(\operatorname{End} V)^{*}\right), \eta_{a b}, \eta_{b a} \in \Omega_{C^{\infty}}^{(1,0)}\left(\mathcal{U}_{a b},(\operatorname{End} V)^{*}\right),
$$




$$
\xi_{a b}, \xi_{b a} \in \Omega_{C^{\infty}}^{(0,1)}\left(\mathcal{U}_{a b},(\operatorname{End} V)^{*}\right) .
$$

We will see that after the symplectic reductions these fields will obey some special constraints. Now we have all initial data to start from the top of the diagram (4.10) -the fields, the symplectic form $\omega_{\Sigma}$ (4.11) and the gauge groups actions (4.13), (4.14),(4.15).

Theorem 4.1 There exist two ways of symplectic reductions represented by the commutative diagram (4.10) which leads from $T^{*} \mathcal{G}_{\Sigma}$ to the cotangent bundle to the moduli space $T^{*} \mathcal{L}$.

To prove Theorem we shall go down along the diagram.

4. Consider first the action of $\mathcal{G}_{\Sigma}^{A}$ (4.16). Let $T^{*} \mathcal{R}_{\Sigma}=\{\Psi, \eta, h\}$ and $h$ is GTF with symplectic form

$$
\omega_{\Sigma}=D\left\{\sum_{a}\left[\int_{\mathcal{U}_{a}} \operatorname{tr}\left(\Psi_{a} D h_{a} h_{a}^{-1}\right)+\sum_{b} \int_{\Gamma_{a}^{b}(C D)} \operatorname{tr}\left(\eta_{a b} D h_{a} h_{a}^{-1}\right)\right]\right\} .
$$

\section{Lemma 4.1}

$$
T^{*} \mathcal{R}_{\Sigma}=T^{*} \mathcal{G}_{\Sigma} / / \mathcal{G}_{\Sigma}^{A}=\mu_{\mathcal{G}_{\Sigma}^{A}}^{-1}(0) / \mathcal{G}_{\Sigma}^{A}
$$

Proof. It follows from (4.11), (4.15) that the Hamiltonian of $\mathcal{G}_{\Sigma}^{A}$ action is

$$
F_{\epsilon^{A}}=\sum_{a>b} F_{a b}, \quad F_{a b}=\int_{\Gamma_{a}^{b}(C D)} \operatorname{tr}\left(\epsilon_{a b}^{A} h_{a}^{-1} \bar{\partial} h_{a}\right)+\int_{\Gamma_{b}^{a}(D C)} \operatorname{tr}\left(\epsilon_{b a}^{A} h_{b}^{-1} \bar{\partial} h_{b}\right) .
$$

In fact the one-form

$$
D F_{a b}=\int_{\Gamma_{a}^{b}(C D)}\left\{\operatorname{tr}\left(\bar{\partial} \epsilon_{a b}^{A} h_{a}^{-1} D h_{a}\right)+\operatorname{tr}\left(\epsilon_{a b}^{A}\left[h_{a}^{-1} \bar{\partial} h_{a}, h_{a}^{-1} D h_{a}\right]\right)\right\}
$$

can be obtain from $\omega_{\Sigma}$ (4.11) by the action of the vector field generated by $\mathcal{G}_{\Sigma}^{A}$ (4.15). But $\epsilon_{a b}^{A}=\epsilon_{b a}^{A}$ (4.15). Putting the moment equal to zero $\mu_{\mathcal{G}_{\Sigma}^{A}}=0$ we come to the constraints $h_{a}^{-1} \bar{\partial} h_{a}=h_{b}^{-1} \bar{\partial} h_{b}$, which are exactly (2.5). Note that the gauge transform (4.16) allows to fix $\xi_{a b}=0$. Thus the symplectic quotient $T^{*} \mathcal{R}_{\Sigma}=T^{*} \mathcal{G}_{\Sigma} / / \mathcal{G}_{\Sigma}^{A}$ has the field content $\left(\Psi, \eta, h \in \Omega_{C^{\infty}}^{0}(\Sigma, \mathcal{P})\right)$ with $\omega_{\Sigma}$ (4.17).

5. Consider the action of $\mathcal{G}_{\Sigma}^{\text {hol }}(2.20),(1.13)$ on $T^{*} \mathcal{R}_{\Sigma}$, which corresponds to the left arrow in the diagram (4.11). We will prove

\section{Lemma 4.2}

$$
T^{*} \mathcal{L}_{\Sigma}^{D}=\mathcal{G}_{\Sigma}^{\text {hol }} \backslash \backslash T^{*} \mathcal{R}_{\Sigma}=\mathcal{G}_{\Sigma}^{\text {hol }} \backslash \mu_{\mathcal{G}_{\Sigma}^{\text {hol }}}^{-1}(0),
$$

where $T^{*} \mathcal{L}_{\Sigma}^{D}$ is defined by (4.1) with the symplectic structure (4.2). 
Proof. From (4.13) and (4.17) we read off the hamiltonian of the gauge fields

$$
F_{\epsilon^{h o l}}=\sum_{a}\left[\int_{\mathcal{U}_{a}} \operatorname{tr}\left(\Psi_{a} \epsilon_{a}^{h o l}\right)+\sum_{b} \int_{\Gamma_{a}^{b}} \operatorname{tr}\left(\eta_{a b} \epsilon_{a}^{h o l}\right)\right] .
$$

On $\mathcal{U}_{a}$ we can put $\Psi_{a}=\bar{\partial}\left(\tilde{\Phi}_{a}+H_{a}\right)$, where $\tilde{\Phi}_{a} \in \Omega_{C^{\infty}}^{(1,0)}\left(\mathcal{U}_{a},(\operatorname{End} V)^{*}\right.$ and $H_{a}$ is an arbitrary element from $\Omega_{\text {hol }}^{(1,0)}\left(\mathcal{U}_{a},(\operatorname{End} V)^{*}\right)\left(\right.$ see $\left.\mathbf{M}^{\prime *}\right)$. Then

$$
F_{\epsilon^{h o l}}=\sum_{a} \sum_{b} \int_{\Gamma_{a}^{b}} \operatorname{tr}\left(\left(\tilde{\Phi}_{a}+H_{a}+\eta_{a b}\right) \epsilon_{a}^{h o l}\right) .
$$

Resolving the moment constraint $\mu_{\mathcal{G}_{\Sigma}^{h o l}}=0$ gives

$$
\eta_{a b}\left(z_{a}, \bar{z}_{a}\right)=-\tilde{\Phi}_{a}\left(z_{a}, \bar{z}_{a}\right)-H_{a}\left(z_{a}\right), z_{a} \in \mathcal{U}_{a b} .
$$

By means of the Stokes theorem $\omega_{\Sigma}$ (4.17) can be transformed to the form

$$
\begin{gathered}
\omega_{\Sigma}=D\left\{\sum_{a}\left[\int_{\mathcal{U}_{a}} \operatorname{tr}\left(\bar{\partial}\left(\tilde{\Phi}_{a}+H_{a}\right) D h_{a} h_{a}^{-1}\right)+\sum_{b} \int_{\Gamma_{a}^{b}} \operatorname{tr}\left(\eta_{a b} D h_{a} h_{a}^{-1}\right)\right]\right\}= \\
-\sum_{a} D \int_{\mathcal{U}_{a}} \operatorname{tr}\left(\left(\tilde{\Phi}_{a}+H_{a}\right) \bar{\partial}\left(D h_{a} h_{a}^{-1}\right)\right) .
\end{gathered}
$$

Let

$$
\phi_{a}=-h_{a}^{-1}\left(\tilde{\Phi}_{a}+H_{a}\right) h_{a} .
$$

Remind that $H_{a}=H_{a}\left(z_{a}\right)$ is an arbitrary holomorphic function on $\mathcal{U}_{a}$. We will choose it in a such way that $\phi_{a}$ becomes a global section in $\Omega_{C \infty}^{(1,0)}\left(\Sigma,(\operatorname{End} V)^{*}\right)$. In other words

$$
\left.\left(\phi_{a}-\phi_{b}\right)\right|_{\Gamma_{a}^{b}}=0 .
$$

In fact, since $g_{a b}=h_{a} h_{b}^{-1}$,

$$
h_{a}\left(\phi_{b}-\phi_{a}\right) h_{a}^{-1}=\left(\tilde{\Phi}_{a}-g_{a b} \tilde{\Phi}_{b} g_{a b}^{-1}\right)+\left(H_{a}-g_{a b} H_{b} g_{a b}^{-1}\right),
$$

where the second term is holomorphic. Consider the integral $I_{a}$ over the contour $\Gamma_{a}=\cup_{b} \Gamma_{a}^{b}$ around $\mathcal{U}_{a}$

$$
I_{a}=-\int_{\Gamma_{a}} \sum_{b} \frac{\left(\tilde{\Phi}_{a}-g_{a b} \tilde{\Phi}_{b} g_{a b}^{-1}\right)(y)}{z-y} d y .
$$

Due to the Sokhotsky-Plejel theorem [13] $I_{a}$ is holomorphic inside and outside $\Gamma_{a}$. It has a jump $\tilde{\Phi}_{a}-g_{a b} \tilde{\Phi}_{b} g_{a b}^{-1}$ on the contour. Let

$$
\begin{gathered}
H_{a}=I_{a} \text { in } \mathcal{U}_{a}, \\
H_{b}=g_{a b}^{-1} I_{a} g_{a b} \text { outside } \mathcal{U}_{a} .
\end{gathered}
$$

Therefore the functions $H_{a}$ and $g_{a b} H_{b} g_{a b}^{-1}$ defining by this integral provide the vanishing of the left hand side (4.21). The symplectic form $\omega_{\Sigma}$ in terms of $\phi$ and $\bar{A}$ can be rewritten as

$$
\omega_{\Sigma}=\sum_{a} D \int_{\mathcal{U}_{a}} \operatorname{tr}\left(\phi_{a} D \bar{A}_{a}\right)=D \int_{\Sigma} \operatorname{tr}(\phi D \bar{A}) .
$$


This form coincides with $\omega^{D}(4.2)$ for $T^{*} \mathcal{L}_{\Sigma}^{D}$. The field $\phi$ is invariant under the $\mathcal{G}_{\Sigma}^{\text {hol }}$ action (4.13). Therefore the symplectic reduction by the gauging $\mathcal{G}_{\Sigma}^{\text {hol }}$ leaves us with the fields $\phi$ and $h$ and the symplectic structure (4.2). In other words $T^{*} \mathcal{L}_{\Sigma} / / \mathcal{G}_{\Sigma}^{\text {hol }}=T^{*} \mathcal{L}_{\Sigma}^{D}$. $\square \mathrm{We}$

can now move down along the left side of diagram (4.11) as it was described in $\mathbf{1}$. and obtain eventually $T^{*} \mathcal{L}$.

It will be instructive to look on relations between two type of dual fields $\eta(4.18)$ and $\phi$ (4.19) that arise after these two consecutive reductions. On the first step we found that $\eta$ are boundary valued forms

$$
\eta_{a b}\left(z_{a}, \bar{z}_{a}\right)=\left.h_{a}^{-1} \phi h_{a}\right|_{\mathcal{U}_{a b}}
$$

Moreover, it follows from $(4.20)$ that

$$
\eta_{a b}\left(z_{a}, \bar{z}_{a}\right)=g_{a b}\left(( z _ { a } , \overline { z } _ { a } ) \eta _ { b a } ( z _ { b } ( z _ { a } ) , \overline { z } _ { b } ( \overline { z } _ { a } ) ) g _ { a b } \left(\left(z_{a}, \bar{z}_{a}\right)^{-1}\right.\right. \text {. }
$$

The second reduction gives $\bar{\partial} \phi+[\bar{A}, \phi]=0$ (see 1.). It is equivalent to $\bar{\partial} \eta=0$, due to (4.22).

6. Now look on the right side of the diagram.

\section{Lemma 4.3}

$$
T^{*} \mathcal{L}_{\Sigma}^{C h}=T^{*} \mathcal{R}_{\Sigma} / / \mathcal{G}_{\Sigma}^{C^{\infty}}=\mu_{\mathcal{G}_{\Sigma}^{C \infty}}^{-1}(0) / \mathcal{G}_{\Sigma}^{C^{\infty}}
$$

where $T^{*} \mathcal{L}_{\Sigma}^{C h}$ is the cotangent bundle (4.4) with $\omega^{C h}$ (4.6).

Proof. The gauge action of $\mathcal{G}_{\Sigma}^{C^{\infty}}$ (4.14) on $T^{*} \mathcal{R}_{\Sigma}$ defines the Hamiltonian (see (4.17))

$$
F_{\epsilon} C^{\infty}=\sum_{a}\left\{\int_{\mathcal{U}_{a}} \operatorname{tr}\left(h_{a}^{-1} \Psi_{a} h_{a} \epsilon_{a}^{C^{\infty}}\right)+\sum_{b} \int_{\Gamma_{a}^{b}} \operatorname{tr}\left(h_{a}^{-1} \eta_{a b} h_{a} \epsilon_{a}^{C^{\infty}}\right)\right\}
$$

Consider the zero level of the moment map

$$
\mu_{\mathcal{G}_{\Sigma}^{C}}^{\infty}: T^{*} \tilde{\mathcal{L}}_{\Sigma} \rightarrow \operatorname{Lie}^{*}\left(\mathcal{G}_{\Sigma}^{C^{\infty}}\right)
$$

From the first terms in (4.24) we obtain

$$
\Psi_{a}=0, a=1, \ldots
$$

This choice of $\Psi$ breaks the invariance with respect to replacements of contours . But if $\bar{\partial} \eta_{a b}=0$ then the exact form of the path $\Gamma_{a}^{b}(C, D)$ is nonessential. Note that this choice is consistent with the definition of $\eta$ (4.20) ( $\eta_{a b}=H_{a}$ in the $\mathcal{G}_{\Sigma}^{\text {hol }}$ reduction). Picking up in the second sum in (4.24) integrals over two neighbor edges we come to the condition

$$
\int_{\Gamma_{a}^{b}} \operatorname{tr}\left(h_{a}^{-1} \eta_{a b} h_{a} \epsilon_{a}^{C^{\infty}}\right)+\int_{\Gamma_{b}^{a}} \operatorname{tr}\left(h_{b}^{-1} \eta_{b a} h_{b} \epsilon_{b}^{C^{\infty}}\right)=0 .
$$

Since $\epsilon^{C^{\infty}} \in \operatorname{Lie}\left(\mathcal{G}_{\Sigma}^{C^{\infty}}\right)$ it is "periodic" $\epsilon_{a}^{C^{\infty}}\left(z_{a}\right)=\epsilon_{b}^{C^{\infty}}\left(z_{b}\left(z_{a}\right)\right)$. It gives the following form of constraints

$$
\left(h_{a}^{-1} \eta_{a b} h_{a}\right)\left(z_{a}\right)=\left(h_{b}^{-1} \eta_{b a} h_{b}\left(z_{b}\left(z_{a}\right)\right)\right), z_{a} \in \mathcal{U}_{a b}
$$


or

$$
\eta_{a b}\left(z_{a}\right)=g_{a b}\left(z_{a}\right) \eta_{b a}\left(z_{b}\left(z_{a}\right)\right) g_{a b}^{-1}\left(z_{a}\right),\left(g_{a b}\left(z_{a}\right)=h_{a}\left(z_{a}, \bar{z}_{a}\right) h_{b}^{-1}\left(z_{b}\left(z_{a}\right), \overline{z_{b}\left(z_{a}\right)}\right)\right),
$$

which is just the twisting property of $\eta$ (4.5). Furthermore, the symplectic form $\omega_{\Sigma}$ (4.17) due to vanishing the field $\Psi$ now is

$$
\omega_{\Sigma}=\sum_{a>b} D\left[\int_{\Gamma_{a}^{b}(C D)} \operatorname{tr}\left(\eta_{a b} D h_{a} h_{a}^{-1}\right)+\int_{\Gamma_{b}^{a}(D C)} \operatorname{tr}\left(\eta_{b a} D h_{b} h_{b}^{-1}\right)\right] .
$$

Taking into account that

$$
\left(D g_{a b} g_{a b}^{-1}\right)\left(z_{a}\right)=D h_{a}\left(z_{a}\right) h_{a}^{-1}\left(z_{a}\right)-h_{a}\left(z_{a}\right)\left(h_{b}^{-1} D h_{b}\right)\left(z_{b}\left(z_{a}\right)\right) h_{a}\left(z_{a}\right)^{-1}
$$

and the moment constraint 4.25 we can rewrite $\omega_{\Sigma}$ as

$$
\omega_{\Sigma}=\sum_{\text {edges }} \int_{\Gamma_{a}^{b}(C D)} D \operatorname{tr}\left(\eta_{a b}\left(z_{a}\right)\left(D g_{a b} g_{a b}^{-1}\right)\left(z_{a}\right)\right) .
$$

It is just $\omega^{C}$ (4.6) in the C-type description of holomorphic bundles. We have the same field content and the same symplectic structure as in $T^{*} \mathcal{L}_{\Sigma}^{C h}$. Therefore $T^{*} \mathcal{R}_{\Sigma} / / \mathcal{G}_{\Sigma}^{C \infty}=T^{*} \mathcal{L}_{\Sigma}^{C h}$.

The last step on the right side of diagram was described in $\mathbf{2}$. Its completes the proof of Theorem.

\section{Schottky description of Hitchin systems}

1. Now consider the last step in the diagram (4.10) in the Schottky parameterization. Since in this case we have only one topologically nontrivial cell $\tilde{\Sigma}$ the symplectic reduction is differ from the described in $\mathbf{4 . 2}$ for the standard covering. In this case the holomorphic fields $\eta_{a}, g_{a}=g_{a}\left(z_{a}\right), a=1, \ldots, g$ live in vicinities $\mathcal{V}_{a}$ of $\mathcal{A}_{a}$-cycles, and $z_{a}$ are local parameters in the internal disks. (see (3.1)). The phase space is

$$
T^{*} \mathcal{L}_{\Sigma}^{C h}=\left\{\eta_{a}, g_{a} \mid \eta_{a} \in \Omega_{h o l}^{(1,0)}\left(\mathcal{V}_{a},(\operatorname{End} V)^{*}\right), g_{a} \in \Omega_{h o l}^{0}\left(\mathcal{V}_{a}, P\right)\right\}
$$

In other words in accordance with (3.3)

$$
T^{*} \mathcal{L}_{\Sigma}^{C h}=\oplus_{a=1}^{g} T^{*} L_{a}(G L(N, \mathbf{C})),
$$

and the loop groups $L_{a}(G L(N, \mathbf{C}))$ are extended by the projective transformations of $z_{a}$ as in (3.3). The symplectic form on this object is (see 4.6)

$$
\omega^{C h}=\sum_{a=1}^{g} D \int_{\mathcal{A}_{a}} \operatorname{tr}\left(\eta_{a}\left(z_{a}\right), D g_{a} g_{a}^{-1}\left(z_{a}\right)\right) .
$$

The gauge transformations (2.16), (4.7) act as the common conjugations by global holomorphic in $\tilde{\Sigma}$ matrix functions

$$
\eta_{a}\left(z_{a}\right) \rightarrow f\left(z_{a}\right) \eta_{a} f^{-1}\left(z_{a}\right), \quad g_{a} \rightarrow f\left(z_{a}\right) g_{a}\left(z_{a}\right) f^{-1}\left(\gamma_{a}\left(z_{a}\right)\right)
$$


The invariant commuting Hamiltonians (4.8) in this parameterization are

$$
I_{j, k}^{C}=\sum_{a} \int_{\mathcal{A}_{a}} \nu_{(j, k)}^{C}\left(z_{a}\right) \operatorname{tr}\left(\eta_{a}^{j}\left(z_{a}\right)\right)
$$

The gauge transform (5.2) produces the moment map $\mu_{\mathcal{G}_{\Sigma}^{\text {hol }}}$, which takes the form

$$
\mu_{\mathcal{G}_{\Sigma}^{\text {hol }}}=\eta_{a}\left(\gamma_{a}\left(z_{a}\right)\right)-\left(g_{a}^{-1} \eta_{a} g_{a}\right)\left(z_{a}\right), a=1, \ldots g
$$

Assume as above that $\mu_{\mathcal{G}_{\Sigma}^{\text {hol }}}=0$ :

$$
\eta_{a}\left(\gamma_{a}\left(z_{a}\right)\right)-\left(g_{a}^{-1} \eta_{a} g_{a}\right)\left(z_{a}\right)=0, a=1, \ldots g
$$

which is twisting property (4.5) in the Schottky picture.

2. The solutions of the moment equations are known in a few degenerate cases 10. We will consider here as an example of the above construction holomorphic bundles over elliptic curves with a marked point.

Define the elliptic curve as the quotient

$$
\Sigma_{\tau}=\mathbf{C}^{*} / q^{\mathbf{Z}}, q=\exp 2 \pi i \tau
$$

In this case

$$
T^{*} \mathcal{L}_{\Sigma}^{C h} \sim(\eta(z), g(z) ; p, s)
$$

where $s \in \mathrm{GL}(N, \mathbf{C})$ is a group element in the marked point $z=1$ and $p \in \operatorname{Lie}^{*}(\mathrm{GL}(N, \mathbf{C}))$. In addition to (5.2)

$$
p \rightarrow f(z) p f^{-1}(1), s \rightarrow f(1) s .
$$

The one form $\eta(z)$ has a pole in the singular point $z=1$. The symplectic form (5.1) on these objects is

$$
\omega^{C h}=D \int_{\mathcal{A}} \operatorname{tr}\left(\eta(z) D g g^{-1}(z)\right)+D \operatorname{tr}\left(p D s s^{-1}\right) .
$$

The transition map $g(z)$ can be diagonalized by (5.2):

$$
g(z)=\exp 2 \pi i \vec{u}=\exp \left\{\operatorname{diag} 2 \pi i\left(u_{1}, \ldots, u_{N}\right)\right\},
$$

where $u_{j}$ are $z$-independent. We keep the same notation for the transformed $\eta(z)=$ $\Sigma_{n \in \mathbf{Z}} \eta_{j, k}^{(n)} z^{n}$. The moment equation (5.5) takes the form

$$
\eta(q z)-\left(g^{-1} \eta g\right)(z)=p \delta(z)
$$

Rewrite it as

$$
q^{n} \eta_{j, k}^{(n)}-e^{2 \pi i\left(x_{k}-x_{j}\right)} \eta_{j, k}^{(n)}=p_{j, k}^{(n)} .
$$

After the resolving the moment constraints we find

$$
\begin{gathered}
\eta_{j, j}(z)=w_{j}, p_{j j}=0 \\
\eta_{j, k}=-\frac{1}{2 \pi i} \frac{\theta\left(u_{j}-u_{k}-\zeta\right) \theta^{\prime}(0)}{\theta\left(u_{j}-u_{k}\right) \theta(\zeta)}, z=\exp 2 \pi i \zeta
\end{gathered}
$$


where $w_{j}$ are new free parameters and $\theta(\zeta)=\sum_{n \in \mathbf{Z}} e^{\pi i\left(n^{2} \tau+2 n \zeta\right)}$. The symplectic form (5.5) on the reduced space takes the form

$$
\omega^{r e d}=D \vec{w} \cdot D \vec{u}+\operatorname{tr} D\left(J s^{-1} D s\right),
$$

and $J$ defines the coadjoint orbit $p=s^{-1} J s$. Consider the quadratic Hamiltonian (5.3). After the reduction $H$ takes the form of the N-body elliptic Calogero Hamiltonian with the spins [14]:

$$
H=\frac{1}{2}\left(\vec{w} \cdot \vec{w}+\frac{1}{4 \pi^{2}} \sum_{j>k}^{N}\left[p_{j, k} p_{k, j} \wp\left(u_{j}-u_{k} \mid \tau\right)+E_{2}(\tau)\right]\right) .
$$

Here $E_{2}(\tau)$ is the normalized Eisenstein series.

Acknowledgments. We would like to thank V.Fock, B.Khesin, N.Nekrasov and A.Rosly for illuminating discussions. We are grateful to the Max Planck Institute for Mathematik in Bonn for the hospitality where this work was prepared. The work of A.L. is supported in part by the grant INTAS 944720 and the grant ISF NSR-300. The work of M.O. is supported in part by the grant CEE-INTAS 932494 and the grant RFFI-96$02-18046$

\section{References}

[1] M.Atyah and R.Bott, The Yang-Mills equations over Riemann surfaces, Phil. Trans. R. Soc. Lond. A308 (1982) 523-615

[2] V.Knizhnik and A.Zamolodchikov, Nucl.Phys. B247 (1984) 83

[3] D.Bernard, Nucl.Phys. B303 (1988) 77; Nucl.Phys. 309 (1988) 145

[4] A.Tsuchia, K.Ueno and Y.Yamada, Adv. Stud. in pure Math. 19 (1989) 459-565

[5] A.Beilinson and V.Schetman, Comm. Math. Phys. 119 (1988) 651-701

[6] A.Presssley and G.Segal, Loop Groups, Claredon Press, Oxford 1986

[7] N.Hitchin, Stable bundles and Integrable Systems, Duke Math. Journ., 54 (1987) 91-114

[8] A.Losev, Coset construction and Bernard equation, Preprint CERN-TH.6215/91

[9] D.Ivanov, KZB equations on Riemann surfaces, hep-th/9410091

[10] N.Nekrasov, Holomorphic bundles and many-body systems, PUPT-1534, hep-th/9503157

[11] P.Etingof and I.Frenkel, Central extension of current groups in two dimensions, Commun. Math. Phys. 165 (1994) 429-444

[12] P.Etingof and B.Khesin, Affine Gelfand-Dickey brackets and holomorphic vector bundles, Geom. and Funct. Anal. 4 (1994), 399-423

[13] N.I.Muskhelishvili, Singuläre Integralgeichungen Academie-Verlag, Berlin (1965)

[14] I.Krichever, O.Babelon, E.Biley and M.Talon, Spin generalization of the Calogero-Mozer system and the matrix KP equation, Preprint LPTHE 94/42 